\title{
Caracterización del daño mecánico de la aorta en condición de hipoxia
}

\author{
Modelamiento y simulación numérica \\ de un ensayo de presurización
}

\author{
Alejandro Bezmalinovic ${ }^{1}$, Eugenio Rivera ${ }^{1}$, Claudio García-Herrera ${ }^{1}$, \\ Diego Celentano $^{2}$, Alejandro González-Candia ${ }^{3}$, Emilio A. Herrera ${ }^{3}$
}

\footnotetext{
${ }^{1}$ Departamento de Ingeniería Mecánica, Universidad de Santiago de Chile, Av. Bdo. O’Higgins 3363, Santiago, Chile.

${ }^{2}$ Departamento de Ingeniería Mecánica y Metalúrgica, Centro de Investigación en Nanotecnología y Materiales Avanzados (CIEN-UC), Pontificia Universidad Católica de Chile, Av. Vicuña Mackenna 4860, Santiago, Chile.

${ }^{3}$ Programa de Fisiopatología, Instituto de Ciencias Biomédicas, Facultad de Medicina, Universidad de Chile, Av. Salvador 486, Providencia, Santiago, Chile.

e-mail: alejandro.bezmalinovic@usach.cl, eugenio.rivera@usach.cl,claudio.garcia@usach.cl,dcelentano@ing.puc.cl, eherrera@med.uchile.cl
}

\section{RESUMEN}

Para evaluar de manera fidedigna el riesgo de ruptura de la aorta - junto a los índices de peligrosidad de enfermedades cardiovasculares u otras condiciones extremas y los efectos de posibles tratamientos - se requiere conocer los mecanismos de daño que conducen a ésta. En este trabajo, se caracteriza el daño mecánico del tejido aórtico en condición de hipoxia, analizando numéricamente su respuesta al ser sujeto a un estado de presurización similar al inducido por un ensayo de acopado hidráulico. El comportamiento mecánico de la pared aórtica, se describe mediante un modelo de material hiperelástico con dos direcciones de isotropía transversal y un modelo de daño isótropo; ambos calibrados experimentalmente, a partir de resultados de ensayos de tracción uniaxial previamente reportados, realizados a muestras de aorta torácica de corderos expuestos a hipoxia hipobárica crónica. Se estudia un grupo tratado con melatonina, en contraste a un grupo control. Una vez calibrado el modelo constitutivo, se evalúa su desempeño en la simulación numérica del ensayo de acopado hidráulico, en la cual se analiza la respuesta cuasi-estática de una estructura - en forma de cuarto de disco, fijada en el perímetro curvo - solicitada fuera de su plano por una presión o fuerza por unidad de superficie, permanentemente normal al área de carga. Los datos experimentales y los resultados de las simulaciones numéricas indican, que un tratamiento con melatonina reduce rigidez de la aorta. Adicionalmente, las presiones asociadas al inicio del daño entregadas por la simulación del ensayo son compatibles con una condición de hipertensión arterial.

Palabras clave: hiperelasticidad, daño mecánico isótropo, pared aórtica, ensayo acopado hidráulico, hipoxia.

\section{ABSTRACT}

In order to reliably assess the rupture-risk of the aorta - along with the hazardousness index of cardiovascular diseases or other extreme conditions, and the effect of possible treatments - it is necessary to know the damage mechanisms that lead to it. In this work, the mechanical damage of hypoxic aortic tissue is characterized, numerically predicting its response when subjected to a bulge-test type of pressurization state. The mechanical behavior of the aortic wall, is described using a hyperelastic material model with two transverse-isotropy directions and an isotropic damage model; both experimentally calibrated, from previously reported uniaxial tensile-test results, performed on thoracic aorta samples of lambs exposed to chronic hypobaric hypoxia. A melatonin-treated group is studied in contrast to a control group. Once the constitutive model is calibrated, its performance is evaluated via the numerical simulation of the bulge-pressurization test; in which the quasistatic response of a quarter-disk shaped structure, fixed along its curved perimeter, and loaded out of its plane by a pressure, or force per unit area permanently normal to the loaded area, its analyzed. 
The experimental data and the results of numerical simulations indicate that a melatonin treatment reduces the stiffness of the aorta. Moreover, the group-wise determined pressures, delivered by the bulge-test simulation and associated with the onset of damage, are compatible with an arterial hypertensive condition.

Keywords: hyperelasticity, isotropic mechanical damage, aortic wall, bulge test, hypoxia.

\section{INTRODUCCIÓN}

La aorta es la arteria de mayor espesor del cuerpo, y corresponde - en una innumerable cantidad de especies terrestres - al conducto suministrante de sangre oxigenada a sus tejidos. La morfología estructural de la pared de arterias, consiste de tres capas o túnicas; la capa más interna, denominada Íntima, seguida por las capas Media y Adventicia. La túnica Media, aquella de mayor espesor y relevancia estructural - cumpliendo un rol principal en la regulación de la presión intravascular - se compone de un arreglo tipo sándwich de elastina, paquetes de fibras de colágeno - cuya orientación y distribución varía entre especies y a lo largo del tronco vascular - y células de músculo liso (SMCs) [1].

Mejorar la comprensión de la respuesta biomecánica de la pared aórtica, posee un impacto fundamental en la expectativa de vida a nivel mundial, dado que la caracterización de su comportamiento mecánico permite determinar cómo la pared aórtica y otros tejidos vivos se ven afectados bajo diversos contextos e incluyendo una serie de fenómenos vinculados a su naturaleza, tales como crecimiento, remodelamiento, activación, daño y ruptura. La experimentación junto a la simulación numérica, permiten evaluar la influencia de patologías cardiovasculares sobre las propiedades biomecánicas de la aorta - por ejemplo, arteriosclerosis y aneurismas, en general de alta mortalidad y desarrollo asintomático - considerando una serie de factores de riesgo, tales como antecedentes del historial del paciente (sexo, edad, fumador, hipertensión, etc.), condiciones externas (baja presión de oxígeno) y/o escenarios extremos (accidente). Así, se concede a la biomecánica y a la mecanobiología, la facultad de aportar a construir sistemáticamente criterios "realistas", orientados por ejemplo, a evaluar el riesgo de realizar intervenciones quirúrgicas, analizando los fenómenos que inciden sobre las propiedades mecánicas durante la reparación o sustitución de vasos enfermos; teniendo como motivación principal, disminuir la incertidumbre en medicina vascular.

En particular, personas gestadas, nacidas y criadas a elevadas altitudes - por sobre $2500 \mathrm{msnm}$ - al exponerse a un ambiente con baja presión de oxígeno, pueden desarrollar una condición denominada "hipoxia hipobárica". La hipoxia es reconocida como la responsable de diferentes tipos de problemas cardiovasculares, respiratorios, hematológicos, metabólicos y neurológicos, los cuales pueden ocurrir a diferentes edades a lo largo del ciclo de vida [2]. En las primeras etapas, se observa un aumento en la frecuencia cardíaca, del gasto cardíaco, de la permeabilidad microvascular, de la disfunción endotelial y de la reactividad vascular irregular [3]. A largo plazo, la frecuencia y el gasto cardíacos tienden a reducirse, mientras que las células del músculo liso y los cardiomiocitos se vuelven hipertróficos $[2,4,5]$. Las alteraciones inducidas por la hipoxia, son concomitantes con el remodelamiento vascular y el endurecimiento arterial [6], los cuales a su vez son asociados con cambios significativos en la microestructura arterial. Aunque el impacto de la hipoxia en las propiedades de la pared aórtica aún no ha sido completamente caracterizado, es claro que el funcionamiento vascular está íntimamente vinculado a las propiedades biomecánicas de la aorta. Para contrarrestar los efectos de la hipoxia y recuperar las funciones vasculares normales, se han estudiado una serie de posibles tratamientos farmacológicos, entre ellos, cinaciguat [7], sildenafil [8], melatonina [5] y péptido natriurético auricular (ANP) [9]. A pesar del relativo éxito de estos tratamientos, su impacto en el comportamiento biomecánico de la pared arterial aún no ha sido completamente caracterizado, siendo necesario evaluar si la mejora funcional está asociada a un cambio en las propiedades biomecánicas del tejido.

Por otra parte, se sabe que el comportamiento mecánico de arterias es no-lineal, anisótropo y viscoelástico [10, 11]. Aunque muchos estudios [12- 17] se han abocado a la hiperelasticidad de paredes arteriales, existe una importante contribución inelástica a su respuesta biomecánica in-vitro, la cual típicamente incrementa al aumentar la deformación y se manifesta como ablandamiento (softening) del material [18, 19]. Si bien los mecanismos intrínsecos aún no son comprendidos del todo, se responsabiliza a un efecto Mullins [20, 21] del ablandamiento, al cual a su vez se le relaciona con micro-mecanismos de daño, tales como de desgarradura (tearing) u otros modos que induzcan deformaciones irreversibles en la matriz extracelular [22]. La caracterización del daño en tejidos blandos, generalmente se ha logrado experimentalmente mediante ensayos, de tracción uniaxial monotónica o cíclica y de presurización, siempre en condiciones ex-vivo; pero esto nunca se ha hecho para estudiar los efectos de la hipoxia crónica y sus tratamientos propuestos.

En particular, el ensayo de acopado hidráulico (del inglés bulge-test), esquematizado en la Figura 1 - en el cual, una muestra cuadrilátera de material, es fijada mediante un anillo al orificio de una cámara y presurizado cuasi-estáticamente por un lado, monitoreándose el abultamiento resultante del tejido inflado - es una 
técnica experimental que ha adquirido creciente relevancia para la cuantificación de propiedades mecánicas del tejido aórtico, debido a su capacidad de reproducir aproximadamente - mediante un estado de deformación biaxial indirecto - las condiciones reales de trabajo de material en condiciones in-vivo, por la acción de la presión intravascular, ejercida por el flujo sanguíneo contra la pared de los vasos sanguíneos, cuya intensidad es variable a lo largo de las fases sistólica y diastólica del ciclo cardíaco.

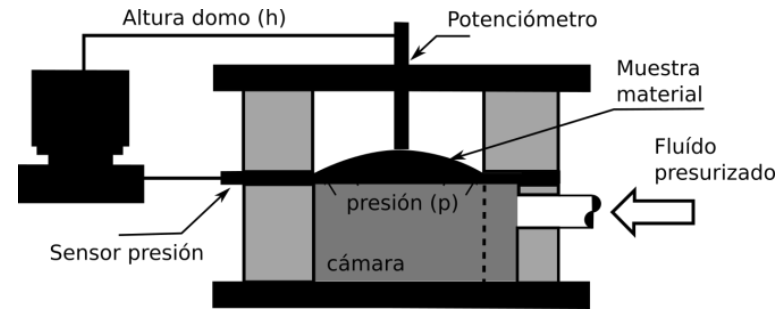

Figura 1: Ensayo de acopado hidráulico, esquema de montaje.

Para evaluar el riesgo de ruptura en tejidos blandos, estudiando los mecanismos de daño que conducen a ésta, se requiere analizar su comportamiento mecánico inelástico. Para ello, son necesarios modelos matemáticos que consideren las deformaciones finitas en las que éstos pueden incurrir, y los efectos del daño mecánico en sus propiedades biomecánicas. La caracterización del daño se ha realizado en arterias y venas [2327], formulando una serie de modelos [20, 21, 28, 29], en general basados en la teoría del daño del continuo (continuum-damage theory), desarrollada inicialmente para elastómeros. En [20] se propone un modelo de daño para tejidos biológicos con una representación de tipo "material compuesto fibroso", donde el daño de las fibras y de la matriz circundante, se rigen por variables separadas. En [21] se introduce un modelo viscoelástico para explicar la histéresis, mientras que [30] considera un modelo de daño con una única variable interna para lesiones en la dirección principal de las fibras, en tanto que [31] extiende los modelos hiperelásticos con ablandamiento, al incluir la energía máxima acumulada requerida para alcanzar la falla. En resumen, los efectos del ablandamiento han sido ampliamente integrados al modelamiento biomecánico de arterias, permitiendo un análisis refinado de la estructura intrínseca basado en la respuesta mecánica del tejido. Curiosamente, estas aplicaciones aún no se han logrado en arterias expuestas a hipoxia crónica.

Por consiguiente, el presente trabajo pretende aplicar un esquema de elementos finitos, para la predicción numérica del daño mecánico en el tejido aórtico en condiciones ex-vivo, sujeto a un estado de presurización similar al inducido por un ensayo de acopado hidráulico. Adicionalmente, se evalúa si un tratamiento con melatonina - una hormona involucrada en la sincronización del ritmo circadiano, incluido el ciclo de sueño-vigilia, la regulación de la presión arterial y su capacidad antioxidante [32, 37, 38, 39] - modifica las propiedades mecánicas elásticas y/o inelásticas de la pared arterial aórtica, desarrollada bajo hipoxia crónica.

\section{MATERIALES Y MÉTODOS}

\subsection{Materiales}

Todos los procedimientos fueron aprobados por el comité de Bioética de la Facultad de Medicina, Universidad de Chile (CBA 0761 FMUCH), y fueron llevados a cabo en concordancia con los lineamientos de llegada y la Guide for the Care and Use of Laboratory Animals, publicada por US National Institutes of Health (NIH Publicación Nro. 85-23, revisada 1996).

Nueve corderos, fueron gestados, nacidos y criados en la estación "Putre", centro de investigación del instituto INCAS (International Center for Andean Studies) de la Universidad de Chile. La estación se ubica en Putre, región de Arica y Parinacota, Chile, a $3600 \mathrm{msnm}$. Los neonatos fueron aleatoriamente separados en dos grupos: 4 tratados con melatonina (MEL, $1,0 \mathrm{mg} \mathrm{kg}^{-1} \mathrm{~d}^{-1}$ de melatonina; vehículo, $0,5 \mathrm{ml} \mathrm{kg}^{-1} \mathrm{~d}^{-1} \mathrm{de}$ Etanol 1,4\%) y 5 controles (CTL, vehículo, $0,5 \mathrm{ml} \mathrm{kg}^{-1} \mathrm{~d}^{-1}$ de Etanol 1,4\%). Ambos tratamientos (MEL y CTL) fueron suministrados por vía oral, cada atardecer, durante 20 días. La eutanasia se realiza alrededor de los 30 días de edad, mediante sobredosis de anestésico (tiopental sódico $100 \mathrm{mg} \mathrm{kg}^{-1}$ i.v.). Una vez extraídas, las aortas se limpian de tejido graso y extra-aórtico, y se preservan en suero fisiológico (krebs) a $4^{\circ} \mathrm{C}$, ensayándose dentro de 24 horas luego de su extracción.

Desde el segmento torácico de cada aorta, se obtienen dos muestras; una con su eje principal orientado según la dirección circunferencial $(\theta)$ del vaso, y la otra con ídem eje orientado según la dirección longitudi- 
nal $(z)$, tal como se indica en la Figura 2. Una vez instaladas en la máquina de ensayo, la geometría inicial de las probetas (aproximadamente rectangular) queda caracterizada por las dimensiones: altura $L_{0}$, ancho $w_{0} \mathrm{y}$ espesor $t_{0}$. La información de las 18 muestras se resume en la Tabla 1.

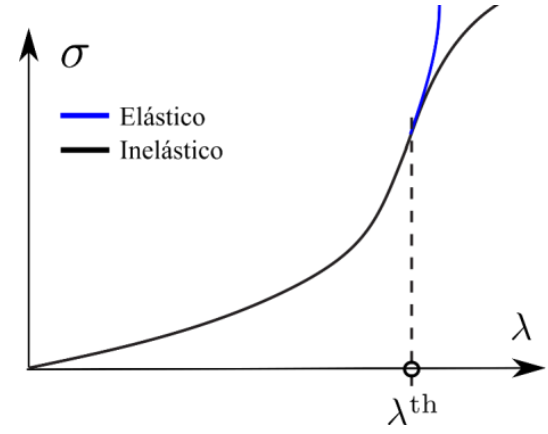

(a)

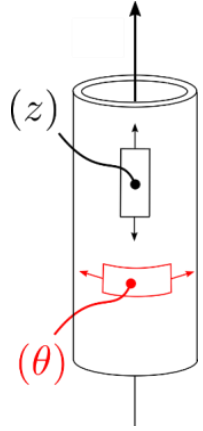

(b)

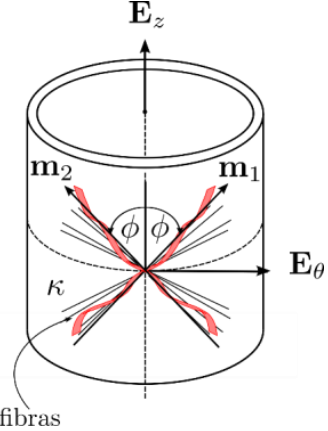

(c)

Figura 2: Curva típica esfuerzo-uniaxial versus alargamiento, casos elástico e inelástico y alargamiento umbral para inicio del daño (a). Esquema probeta orientada según direcciones longitudinal $(z)$ y circunferencial $(\theta)$ (b). Esquema de modelo hiperelástico con dos direcciones de isotropía transversal simétricas con respecto a dirección longitudinal (c).

Tabla 1: Dimensiones promedio probetas (promedio \pm desviación estándar mediciones; en $\mathrm{mm}$ ) por grupo y por orientación, longitudinal $(z)$ o circunferencial $(\theta)$.

\begin{tabular}{c|c|c|c|c|c|c}
\hline $\begin{array}{c}\text { PROBETA } \\
\mathbf{N}^{\circ}\end{array}$ & $\begin{array}{c}\text { SUJETO } \\
\mathbf{N}^{\circ}\end{array}$ & $\begin{array}{c}\text { ALTURA } \\
L_{0}(\mathrm{~mm})\end{array}$ & $\begin{array}{c}\text { ANCHO } \\
w_{0}(\mathrm{~mm})\end{array}$ & $\begin{array}{c}\text { ESPESOR } \\
t_{0}(\mathrm{~mm})\end{array}$ & GRUPO & TIPO \\
\hline 1 & $01-16$ & $6,592 \pm 0,052$ & $6,302 \pm 0,076$ & $2,277 \pm 0,059$ & MEL & $z$ \\
\hline 2 & $01-16$ & $5,384 \pm 0,057$ & $6,200 \pm 0,000$ & $1,908 \pm 0,064$ & MEL & $\theta$ \\
\hline 3 & $02-16$ & $7,612 \pm 0,053$ & $6,805 \pm 0,070$ & $1,688 \pm 0,066$ & CTL & $z$ \\
\hline 4 & $02-16$ & $7,158 \pm 0,034$ & $5,276 \pm 0,103$ & $1,231 \pm 0,031$ & CTL & $\theta$ \\
\hline 5 & $04-16$ & $7,380 \pm 0,039$ & $6,804 \pm 0,077$ & $1,828 \pm 0,056$ & MEL & $z$ \\
\hline 6 & $04-16$ & $6,357 \pm 0,049$ & $6,978 \pm 0,082$ & $2,200 \pm 0,048$ & MEL & $\theta$ \\
\hline 7 & $05-16$ & $7,126 \pm 0,124$ & $7,380 \pm 0,245$ & $2,380 \pm 0,018$ & MEL & $z$ \\
\hline 8 & $05-16$ & $7,196 \pm 0,064$ & $6,079 \pm 0,102$ & $2,394 \pm 0,089$ & MEL & $\theta$ \\
\hline 9 & $06-16$ & $4,478 \pm 0,040$ & $7,949 \pm 0,178$ & $1,817 \pm 0,033$ & CTL & $z$ \\
\hline 10 & $06-16$ & $4,842 \pm 0,064$ & $6,497 \pm 0,067$ & $2,753 \pm 0,028$ & CTL & $\theta$ \\
\hline 11 & $08-16$ & $6,326 \pm 0,048$ & $6,727 \pm 0,057$ & $1,362 \pm 0,028$ & CTL & $z$ \\
\hline 12 & $08-16$ & $6,706 \pm 0,038$ & $5,079 \pm 0,190$ & $1,479 \pm 0,039$ & CTL & $\theta$ \\
\hline 13 & $13-16$ & $6,946 \pm 0,037$ & $4,419 \pm 0,181$ & $2,496 \pm 0,043$ & MEL & $z$ \\
\hline 14 & $13-16$ & $7,223 \pm 0,049$ & $5,074 \pm 0,041$ & $2,523 \pm 0,050$ & MEL & $\theta$ \\
\hline 15 & $15-16$ & $6,843 \pm 0,037$ & $3,729 \pm 0,107$ & $2,609 \pm 0,045$ & CTL & $z$ \\
\hline 16 & $15-16$ & $6,480 \pm 0,057$ & $5,773 \pm 0,028$ & $2,810 \pm 0,045$ & CTL & $\theta$ \\
\hline 17 & $16-16$ & $6,386 \pm 0,077$ & $5,321 \pm 0,101$ & $2,557 \pm 0,085$ & CTL & $z$ \\
\hline 18 & $16-16$ & $6,329 \pm 0,073$ & $5,691 \pm 0,107$ & $1,741 \pm 0,037$ & CTL & $\theta$ \\
\hline
\end{tabular}

\subsection{Ensayos de tracción uniaxial}

Cada muestra es sujeta, sin pre-condicionamiento, a un ensayo de tracción uniaxial con desplazamiento controlado, empleando una máquina de ensayo mono-columna (Instron 3342).

La fuerza axial $F$, es medida mediante una celda de carga con capacidad de $10 \mathrm{~N}$, y se emplea una tasa de carga de $1 \mathrm{~mm} / \mathrm{min}$, hasta alcanzar la rotura. Durante cada ensayo, la muestra es inmersa en un buffer fosfato salino (PBS $1 \mathrm{x})$ a $39^{\circ} \mathrm{C}$. 
Las curvas de respuesta, obtenidas en formato fuerza versus desplazamiento, pueden convertirse en curvas de esfuerzo-alargamiento $(\sigma-\lambda)$, con alargamiento $\lambda=1+\Delta L\left(L_{0}\right)^{-1}$, y con esfuerzo real o de Cauchy $\sigma=F \lambda\left(w_{0} h_{0}\right)^{-1}$. Una curva típica esfuerzo-uniaxial versus alargamiento, se muestra en la Figura 2a.

\subsection{Modelo constitutivo}

En concordancia con la respuesta observada en las muestras de material aórtico ensayadas - curva tipo "J" con un cambio de curvatura - para predecir el comportamiento del material se asume un modelo constitutivo hiperelástico el cual incluye un modelo de daño isótropo.

El empleo de modelos hiperelásticos implica la definición de una función "densidad de energía de deformación elástica" (SEF), $\Psi: \mathbb{S}(3) \times[0,1] \rightarrow \mathbb{R}$, la cual es descompuesta aditívamente, en una componente dilatacional y otra distorsional o isocórica [25,33], siendo esta última y por hipótesis, aquella afectada por el daño [20]. Adicionalmente, se asume que la componente isocórica de la función de energía, corresponde a la suma de tres términos, representantes de la contribución de una matriz blanda y de dos familias de fibras reforzantes. Eventualmente, la SEF puede escribirse:

$$
\Psi(\mathbf{C}, \mathrm{D})=\Psi_{\mathrm{vol}}(J)+(1-\mathrm{D})\left[\Psi_{m}^{0}(\overline{\mathbf{C}})+\Psi_{f, 1}^{0}(\overline{\mathbf{C}})+\Psi_{f, 2}^{0}(\overline{\mathbf{C}})\right],
$$

donde $\Psi_{\mathrm{vol}}$ es la contribución dilatacional, mientras que, $\Psi_{m}^{0}, \Psi_{f, 1}^{0}$ y $\Psi_{f, 2}^{0}$, son las contribuciones vírgenes o sin daño, de la matriz y de ambas familias de fibras, y $\mathrm{D}: \mathbb{R}_{+} \rightarrow[0,1]$ es una variable interna de daño.

Adicionalmente, $\mathbf{C}=\mathbf{F}^{\mathrm{T}} \mathbf{F} \in \mathbb{S}(3)$ denota a la deformación de Cauchy-Green por la derecha, con $\mathbf{F} \in \mathbb{G} \mathbb{L}(3)$ el gradiente de deformación, $J=\operatorname{det}(\mathbf{F})$, y $\overline{\mathbf{C}}=J^{-2 / 3} \mathbf{C}$ la componente isocórica de la deformación, definida tal que $\operatorname{det}(\overline{\mathbf{C}})=1$.

La componente de energía para la matriz, correspondiente a un modelo tipo Neo-Hooke, puede escribirse $\Psi_{m}^{0}=C_{1}(\overline{\mathbf{C}}: \mathbf{1}-\mathbf{3}) / 2$, donde $C_{1}$ es una constante de material, mientras que la contraparte asociada a la k-ésima familia de fibras es:

$$
\Psi_{f, k}^{0}=\frac{C_{2}}{2 C_{3}}\left\{\exp \left(C_{3}\left[\kappa(\overline{\mathbf{C}}: \mathbf{1})+(1-3 \kappa) \overline{\mathbf{C}}: \mathbf{M}_{\mathrm{k}}-1\right]^{2}\right)-1\right\},
$$

donde $C_{2}, C_{3}$, y $\kappa$ son constantes de material, $\mathbf{M}_{\mathrm{k}}=\mathbf{m}_{\mathrm{k}} \otimes \mathbf{m}_{\mathrm{k}}$ es un "tensor estructural", con $\mathbf{m}_{\mathrm{k}}=$ $\sin \left(\varphi_{k}\right) \mathbf{E}_{\theta}+\cos \left(\varphi_{k}\right) \mathbf{E}_{\mathrm{z}}$ el versor de la k-ésima familia de fibras, escrito en términos de los vectores unitarios, $\mathbf{E}_{\theta}$ y $\mathbf{E}_{\mathrm{z}}$, que respectivamente definen a las direcciones circunferencial y longitudinal del material, considerando $\varphi_{k}=\operatorname{acos}\left(\mathbf{m}_{\mathrm{k}} \cdot \mathbf{E}_{\mathrm{z}}\right)$. Para dos familias de fibras, simétricamente alineadas con respecto a la dirección longitudinal, $\varphi_{1,2}= \pm \varphi$, caso mostrado esquemáticamente en la Figura $2 \mathrm{c}$.

A modo de completar el modelo constitutivo, debe determinarse la ecuación de evolución de la variable de daño. Para este propósito, se introduce la "tasa de liberación de energía":

$$
S(t)=\sqrt{2\left[\Psi_{m}^{0}(\overline{\mathbf{C}}(\mathrm{t}))+\Psi_{f, 1}^{0}(\overline{\mathbf{C}}(\mathrm{t}))+\Psi_{f, 2}^{0}(\overline{\mathbf{C}}(\mathrm{t}))\right]},
$$

junto al criterio de daño, $\Phi=S(t)-S^{\text {th }} \leq 0, \forall t$. Así, $\Phi=0$ define, en el espacio de deformaciones isocóricas, a una superficie de daño de radio $S^{\text {th }}=\max (S(\tau)), \tau \in(-\infty, t]$, con vector normal $\mathbf{N}=\partial \Phi / \partial \overline{\mathbf{C}}$.

Luego, se selecciona como función de daño, a la curva [21]:

$$
\mathrm{D}(S)= \begin{cases}1-\exp \left\{\alpha\left(S_{0}^{\text {th }}-S\right)\right\}, & \text { si } S \geq S_{0}^{\text {th }} \\ 0, & \text { si } S<S_{0}^{\text {th }}\end{cases}
$$

donde $S_{0}^{\text {th }}$ es un umbral para el inicio del daño, y $\alpha$ es una constante del material.

La consistencia termodinámica del modelo de daño es establecida, invocando dentro del sólido una tasa no-negativa de producción de entropía por unidad de volumen, por la temperatura absoluta. Despreciando fuerzas másicas y transferencia de calor, la "disipación" se define mediante la desigualdad de Clausius-Duhem [10], $\mathfrak{D}=\dot{w}-\dot{\Psi} \geq 0$, donde $\dot{w}=\mathbf{S}: \dot{\mathbf{C}} / 2$, es la tasa de trabajo interno por unidad de volumen sin deformar, con $\mathbf{S}=2(\partial \Psi / \partial \mathbf{C}) \in \mathbb{S}(3)$, el esfuerzo de Piola-Kirchhoff.

Dado que, $\dot{\Psi}=(\partial \Psi / \partial \mathbf{C}): \dot{\mathbf{C}}+(\partial \Psi / \partial \mathrm{D}) \dot{\mathrm{D}}$, es posible definir una "fuerza termodinámica": 


$$
f=-\frac{\partial \Psi}{\partial \mathrm{D}}=\Psi_{f, 1}^{0}+\Psi_{f, 2}^{0} \geq 0
$$

Considerando la condición de consistencia, $\Phi=\dot{\Phi}=0$, y si $\dot{\overline{\mathbf{C}}}$ es la tasa de deformación isocórica, la evolución del daño es controlada por la ecuación irreversible [20]:

$$
\dot{\mathrm{D}}=\left\{\begin{array}{c}
\frac{\mathrm{dD}}{\mathrm{d} S} \dot{S}, \text { si } \Phi=0 \wedge \mathbf{N}: \dot{\overline{\mathbf{C}}}>0 \\
0, \quad \text { en otro caso }
\end{array}\right.
$$

Finalmente, el esfuerzo de Cauchy es, $\boldsymbol{\sigma}=J^{-1} \mathbf{F} \mathbf{S} \mathbf{F}^{\mathrm{T}} \in \mathbb{S}(3)$.

\subsection{Ajuste de curvas}

Para la configuración de un ensayo de tracción uniaxial, a un sólido compuesto por el material descrito en la sección precedente, si $\lambda_{1}=\lambda$ y $\lambda_{2}$ es uno de los alargamientos de la sección transversal, el esfuerzo teórico es:

$$
\tilde{\sigma}(\lambda)=(1-D)\left\{C_{1}\left(\lambda_{1}^{2}-\lambda_{1}^{-2} \lambda_{2}^{-2}\right)+4 C_{2}\left[\kappa\left(\lambda_{1}^{2}-\lambda_{1}^{-2} \lambda_{2}^{-2}\right)+\lambda_{1}^{2}(1-3 \kappa) \cos ^{2}(\vartheta)\right] K \exp \left(C_{3} K^{2}\right)\right\},
$$

con $K=I_{1} \kappa+(1-3 \kappa) I_{4}-1, I_{1}=\lambda_{1}^{2}+\lambda_{2}^{2}+\left(\lambda_{1} \lambda_{2}\right)^{-2}$, e $I_{4}=\lambda_{1}^{2} \cos ^{2}(\vartheta)+\lambda_{2}^{2} \sin ^{2}(\vartheta) ;$ donde $\vartheta=\varphi$ para probetas cargadas en la dirección longitudinal, y $\vartheta=(\pi / 2-\varphi)$ para probetas cargadas en la dirección circunferencial.

En virtud de lo mostrado por los experimentos, el daño se desarrolla de distinta manera sobre las direcciones longitudinal $(z)$ y circunferencial $(\theta)$; hecho atribuible, a que la interacción entre ambas familias de fibras difiere sobre cada dirección de carga. Para incorporar dicho fenómeno, se separa el efecto del daño haciendo:

$$
1-\mathrm{D}=\left(1-\mathrm{D}_{z}\right)\left(1-\mathrm{D}_{\theta}\right)
$$

donde $\mathrm{D}_{z} \mathrm{y}_{\theta}$, son funciones escalares independientes, para cuantificar de forma separada el daño sobre cada dirección, asociadas respectivamente a los parámetros $\alpha_{z}$ y $\alpha_{\theta}$, y a los umbrales, $S_{0, z}^{\text {th }}=S\left(\lambda_{z}^{\text {th }}\right)$ y $S_{0, \theta}^{\text {th }}=S\left(\lambda_{\theta}^{\text {th }}\right)$, donde $\lambda_{z}^{\text {th }}$ y $\lambda_{\theta}^{\text {th }}$ son alargamientos que marcan la transición entre el rango elástico e inelástico de deformación uniaxial, tal como indica la Figura 2a.

A partir de la ecuación (8), se tienen las propiedades de daño "efectivas" o equivalentes, $\alpha=\alpha_{z}+\alpha_{\theta}$, y $S_{0}^{\text {th }}=\left(\alpha_{z} S_{0, z}^{\text {th }}+\alpha_{\theta} S_{0, \theta}^{\text {th }}\right) \alpha^{-1}$.

La identificación del vector de parámetros, $\xi=\left\{C_{1}, C_{2}, C_{3}, \kappa, \varphi, \alpha_{z}, \alpha_{\theta}, S_{0, z}^{\text {th }}, S_{0, \theta}^{\text {th }}\right\}$, es lograda ajustando el valor teórico dado por ecuación (7), a la información experimental $\sigma(\lambda)$. Dados los datos experimentales de esfuerzo longitudinal $\sigma_{z}$, y circunferencial $\sigma_{\theta}$, el procedimiento de ajuste se formula como la minimización de la función de costo:

$$
\mathcal{J}(\xi)=\left\|\tilde{\sigma}_{z}(\lambda)-\sigma_{z}\right\|^{2}+\left\|\tilde{\sigma}_{\theta}(\lambda)-\sigma_{\theta}\right\|^{2},
$$

realizado, mediante la implementación en lenguaje Fortran, del algoritmo de Levenberg-Marquardt [34]. En cada caso, para un esfuerzo promedio $\bar{\sigma}$, la fidelidad del ajuste, entregado por las propiedades de material identificadas, se evalúa mediante el coeficiente de determinación:

$$
R^{2}=1-\left\{\sum_{i}^{n}\left[\sigma_{i}-\tilde{\sigma}\left(\lambda_{i}\right)\right]^{2}\right\}\left[\sum_{i}^{n}\left(\sigma_{i}-\bar{\sigma}\right)^{2}\right]^{-1}
$$

Las curvas experimentales consideradas para el ajuste de parámetros corresponden a las otorgadas por un promedio simple - punto-a-punto sobre un mismo nivel de alargamiento - de las curvas esfuerzoalargamiento de ensayos individuales, asociadas a probetas de un mismo grupo y orientación.

\subsection{Simulación numérica ensayo de acopado hidráulico}

El problema de interés consiste en analizar una estructura en forma de disco, compuesta de los materiales previamente caracterizados (tipo MEL y tipo CTL), al ser solicitada por condiciones de carga análogas a las de un ensayo de acopado hidráulico.

Se consideran las propiedades de daño "efectivas" siguientes; para el grupo MEL, $\alpha=0,196 \mathrm{kPa}^{-1 / 2}$ y $S_{0}^{\text {th }}=4,09 \mathrm{kPa}^{1 / 2}$, y para el grupo CTL, $\alpha=0,237 \mathrm{kPa}^{-1 / 2}$, y $S_{0}^{\text {th }}=5,19 \mathrm{kPa}^{1 / 2}$. 
La estructura, originalmente de diámetro $L_{0}=9 \mathrm{~mm}$ y espesor $t_{0}$, e impedida de desplazarse en todo su perímetro, se encuentra solicitada sobre la cara $X_{3}=0$, por una presión cuasi-estática - fuerza distribuida $p$, actuante fuera del plano $X_{1} X_{2}$ y siempre normal a la superficie de contacto, denominada "carga seguidora"; un caso particular de fuerza externa dependiente de la deformación.

Se seleccionan los espesores promedio: $t_{0}=2,0 \mathrm{~mm}$ para el grupo CTL, y $t_{0}=2,3 \mathrm{~mm}$ para el grupo MEL. Por la simetría geométrica y de carga presente, es suficiente estudiar un cuarto de la estructura completa, de acuerdo con lo ilustrado en la Figura $3 \mathrm{a}$.

Las simulaciones numéricas contemplan una discretización espacial estándar, mediante 4896 elementos finitos (EF) hexaédricos de ocho nodos, con 5913 nodos en total. La malla y sus condiciones de borde se ilustran en las Figuras $3 a$ y $3 b$.

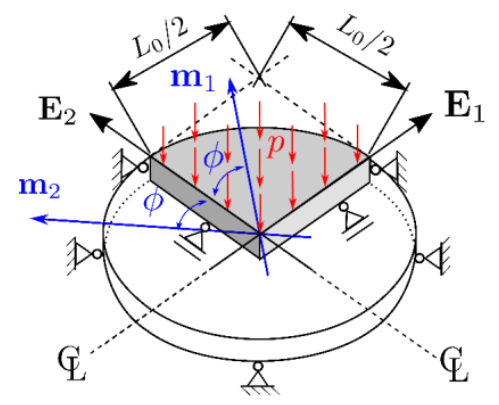

(a)

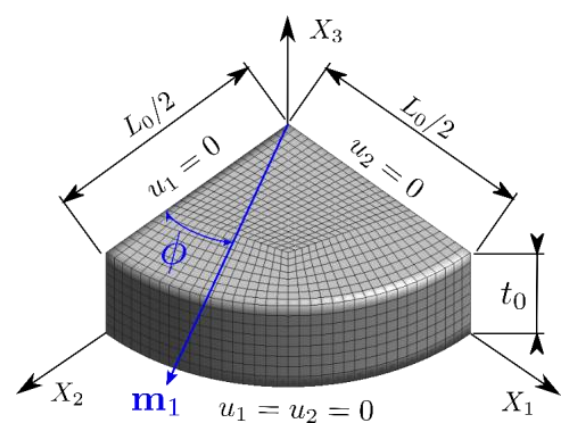

(b)

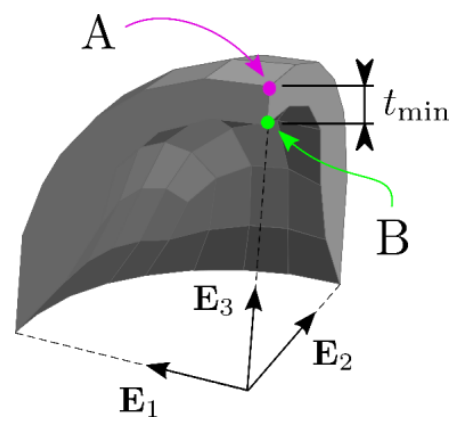

(c)

Figura 3: Esquema simulación ensayo de acopado hidráulico. Estructura reducida por simetrías geométricas y de carga en configuración sin deformar direcciones de isotropía transversal en planta (a); malla de elementos finitos (b); configuración deformada tipo, con espesor mínimo de la estructura y puntos de análisis A y B (c).

\section{RESULTADOS}

\subsection{Curvas experimentales}

Las curvas esfuerzo-alargamiento de ensayos individuales de ambos grupos (MEL y CTL), agrupadas por dirección $(z$ ó $\theta$ ) junto a su correspondiente curva "media" (promedio \pm desviación estándar muestras), se muestran en las Figuras 4 y 5.
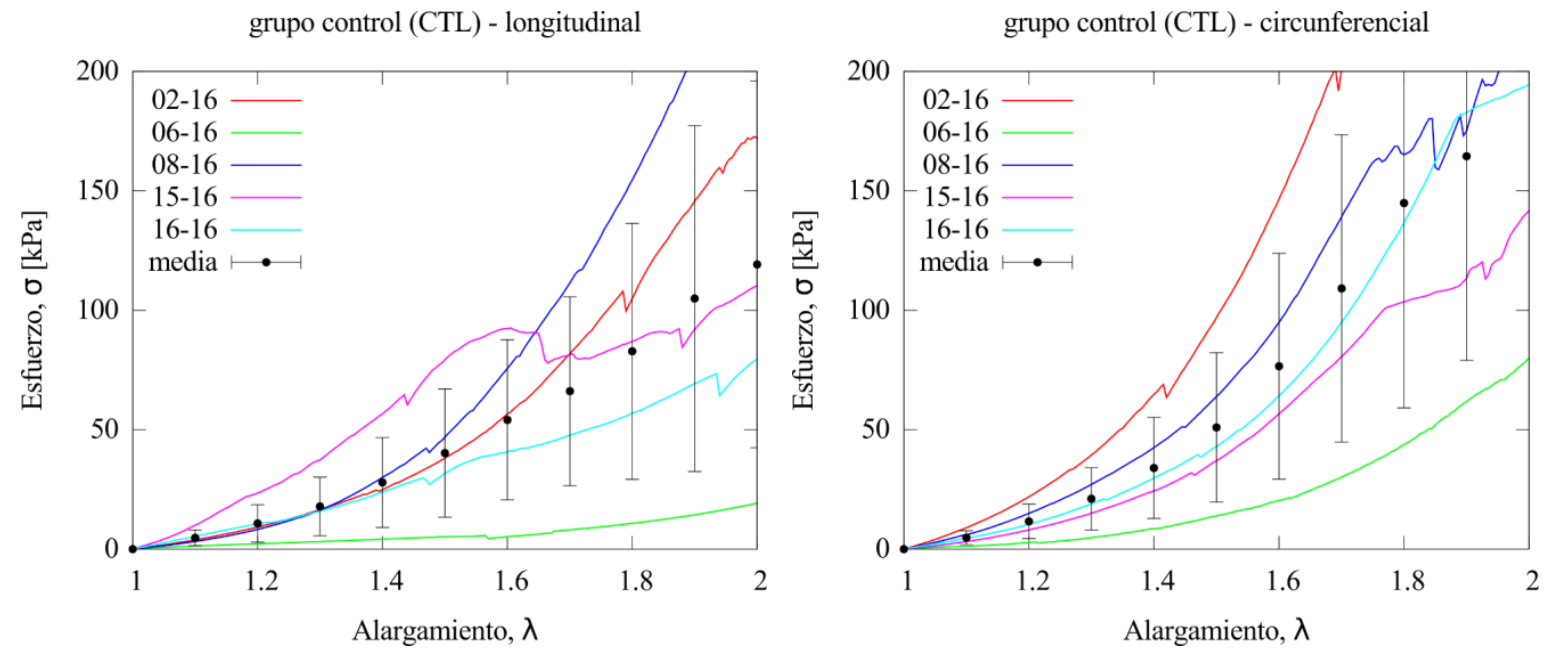
(a)

(b)

Figura 4: Curvas experimentales, esfuerzo uniaxial (en $\mathrm{kPa}$ ) versus alargamiento, grupo control (CTL). Curvas de probetas individuales y curva "media", direcciones longitudinal (a) y circunferencial (b).

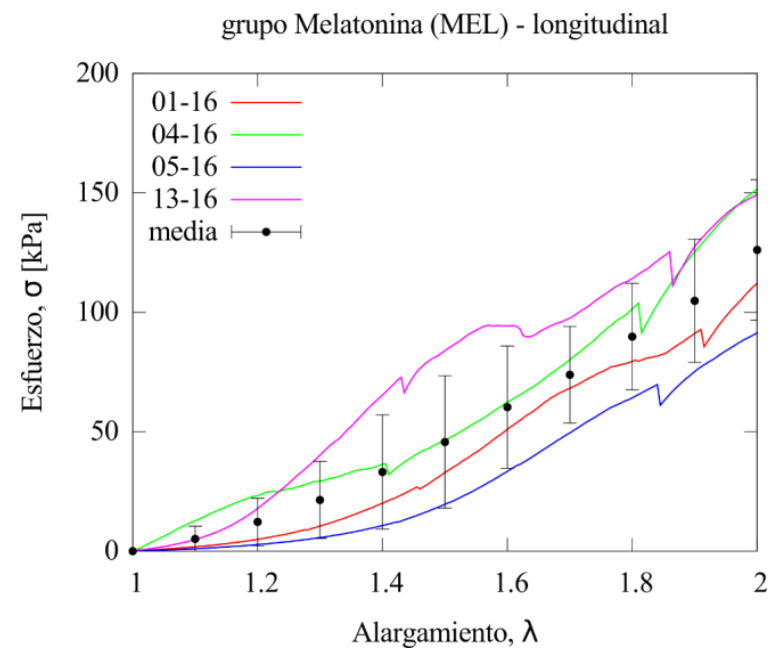

(a)

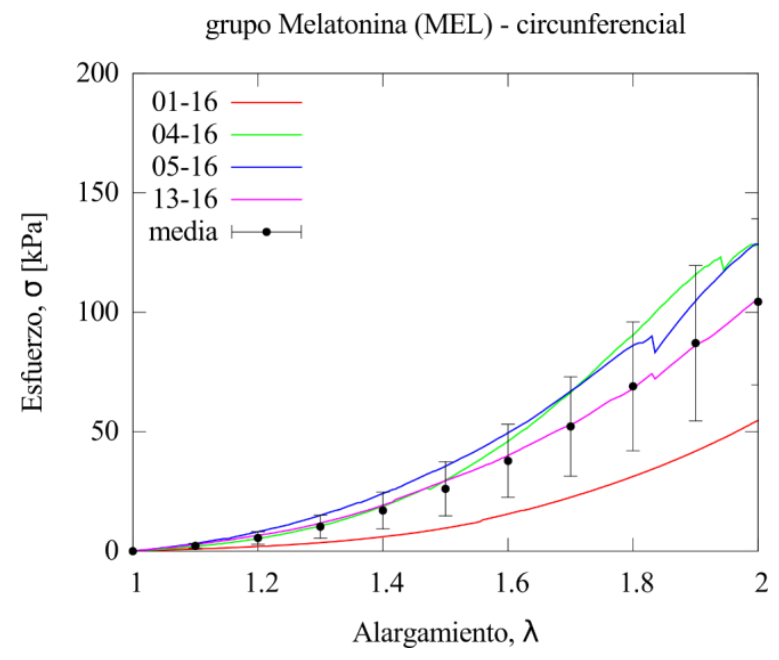

(b)

Figura 5: Curvas experimentales, esfuerzo uniaxial (en $\mathrm{kPa}$ ) versus alargamiento, grupo melatonina (MEL). Curvas de probetas individuales y curva "media", direcciones longitudinal (a) y circunferencial (b).

\subsection{Ajuste del modelo constitutivo}

Los modelos de material determinados para ambos grupos, control (CTL) y tratado con melatonina (MEL), cuyos parámetros son identificados a través del ajuste de curvas, se reportan en la Tabla 2 y se muestran gráficamente, en las Figuras $6 \mathrm{a}$ y $6 \mathrm{~b}$ respectivamente.

Tabla 2: Resumen de ajustes del modelo constitutivo. Constantes identificadas por grupo y agrupadas por dirección; alargamientos de transición al rango inelástico $\lambda^{\text {th }}$, propiedades inelásticas y coeficientes de determinación.

\begin{tabular}{|c|c|c|c|c|c|c|c|c|c|c|c|c|c|}
\hline GRUPO & $\begin{array}{c}\varphi \\
(\mathrm{deg})\end{array}$ & $\begin{array}{c}C_{1} \\
(\mathrm{kPa})\end{array}$ & $\begin{array}{c}C_{2} \\
(\mathrm{kPa})\end{array}$ & $C_{3}$ & $\kappa$ & $\lambda_{z}^{\text {th }}$ & $\lambda_{\theta}^{\text {th }}$ & $\begin{array}{c}\alpha_{z} \\
\left(\mathrm{kPa}^{-1 / 2}\right)\end{array}$ & $\begin{array}{c}\alpha_{\theta} \\
\left(\mathrm{kPa}^{-1 / 2}\right)\end{array}$ & $\begin{array}{c}S_{0, z}^{\mathrm{th}} \\
\left(\mathrm{kPa}^{1 / 2}\right)\end{array}$ & $\begin{array}{c}S_{0, \theta}^{\text {th }} \\
\left(\mathrm{kPa}^{1 / 2}\right)\end{array}$ & $R_{z}^{2}$ & $R_{\theta}^{2}$ \\
\hline & 35,00 & 32 & 27,71 & 0,014 & 0,24 & 1,50 & 1,75 & 0110 & 0086 & 3 & 47 & & 08 \\
\hline CTL & 48,84 & 14,66 & 29,92 & 0,487 & 0,23 & 1,50 & 1,78 & 0,107 & 0,130 & 3,4 & 6,61 & 0,999 & 0,998 \\
\hline
\end{tabular}

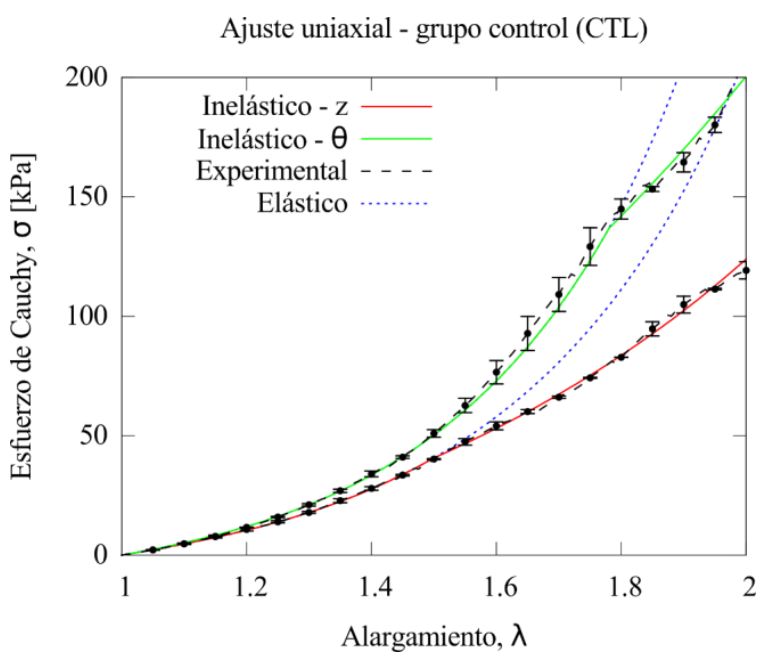

(a)

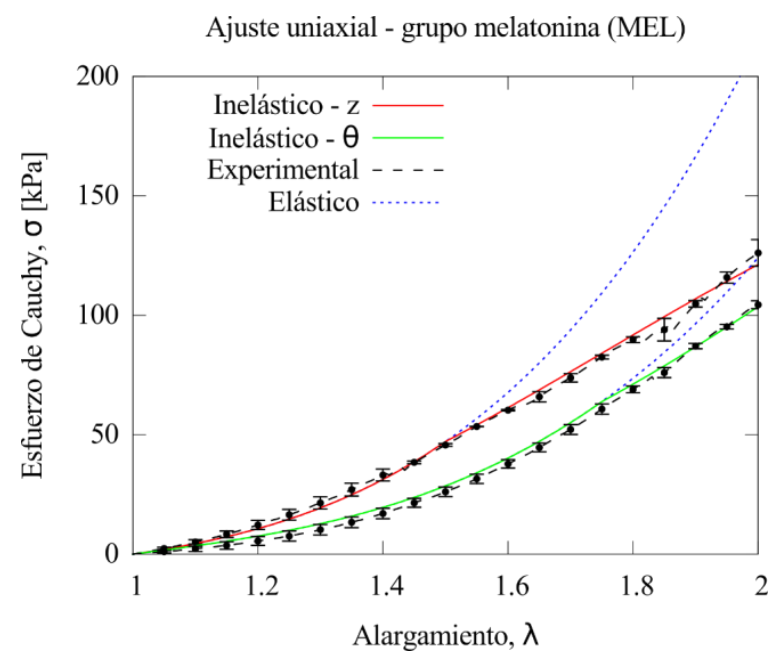

(b) 
Figura 6: Ajuste modelo constitutivo, grupo control (CTL) (a) y grupo melatonina (MEL) (b). Curvas esfuerzo uniaxial (en $\mathrm{kPa}$ ) versus alargamiento, direcciones longitudinal $(z)$ y circunferencial $(\theta)$. Esfuerzo experimental, teóricos elástico e inelástico y error (en $\mathrm{kPa}$ ) esfuerzo teórico inelástico.

\subsection{Simulación numérica ensayo de acopado hidráulico}

En las Figuras 7 y 8, se muestra la evolución - con la presión aplicada - del desplazamiento vertical y las variables internas, sobre los puntos superior (A) e inferior (B) de la arista recta del modelo indicados en la Figura 7a.

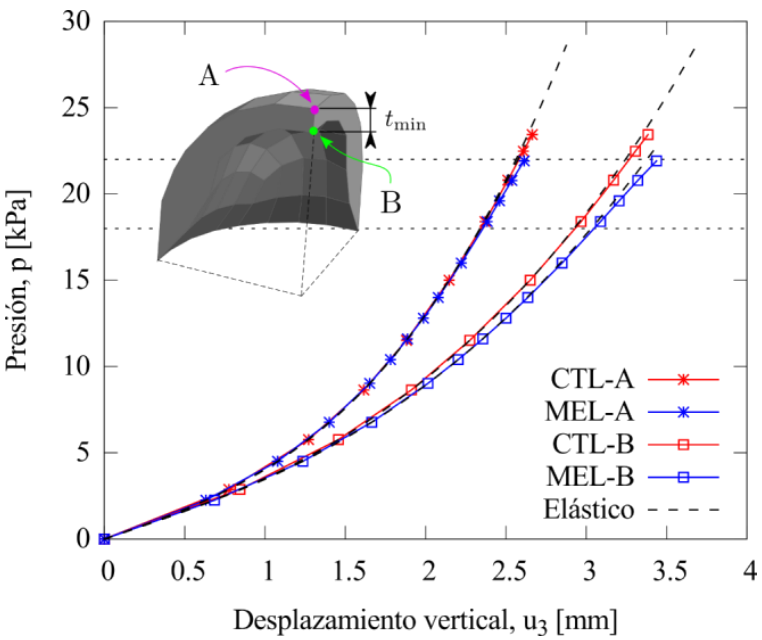

(a)

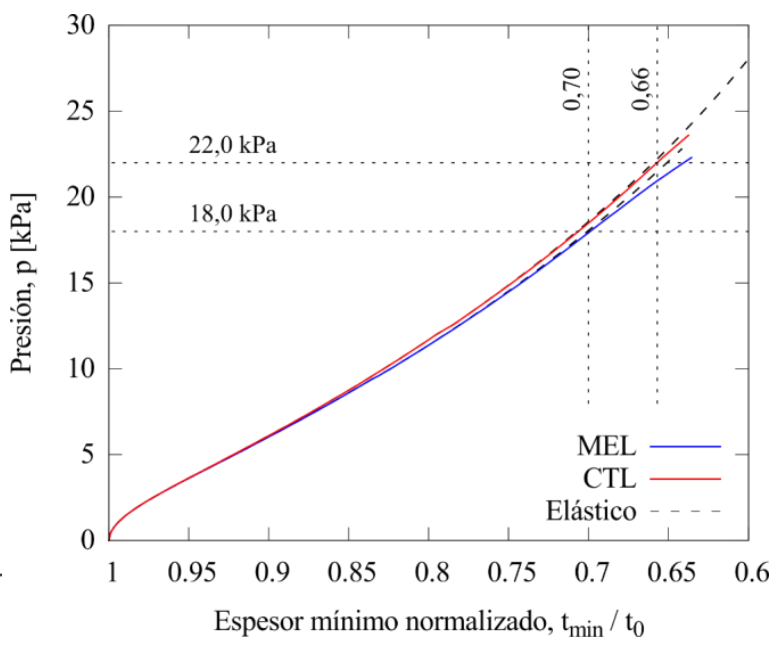

(b)

Figura 7: Simulación ensayo de acopado hidráulico. Curvas presión (en $\mathrm{kPa}$ ), versus desplazamiento vertical en puntos A y B (en mm) (a) y versus espesor mínimo normalizado de la estructura (b). Modelos constitutivos ajustados a grupos melatonina (MEL) y control (CTL). Casos elásticos e inelásticos.

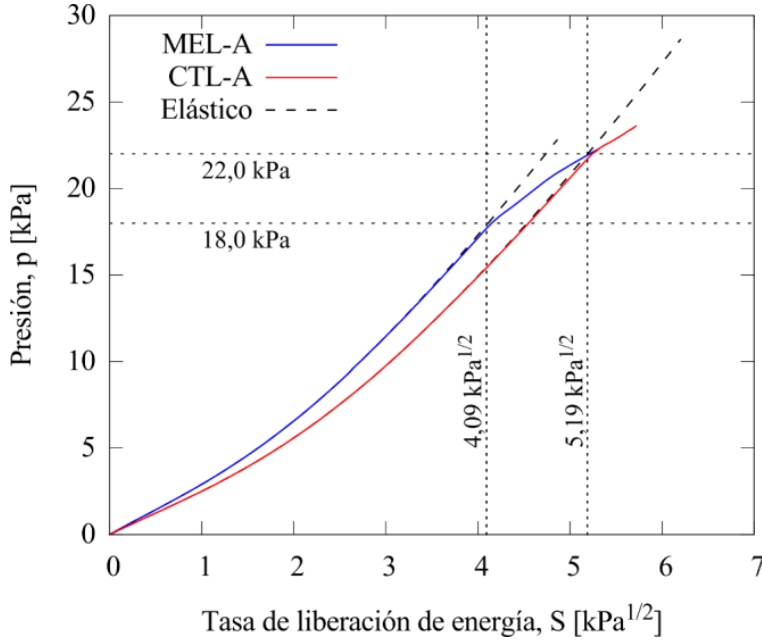

(a)

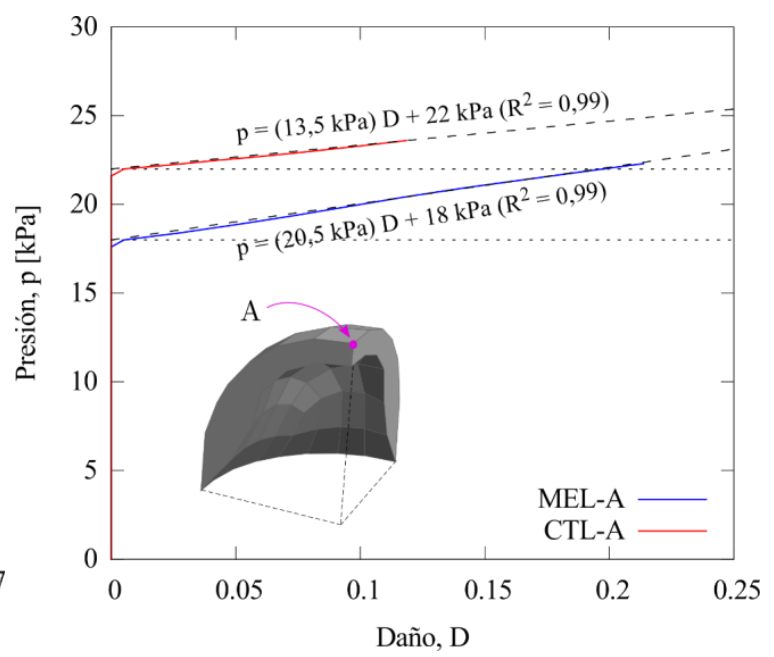

(b)

Figura 8: Simulación ensayo de acopado hidráulico. Curvas presión (en kPa), versus tasa de liberación de energía por daño (en $\mathrm{kPa}^{1 / 2}$ ) (a) y versus daño (b), en punto A. Modelos constitutivos ajustados a grupos melatonina (MEL) y control (CTL). Casos elásticos e inelásticos.

\section{DISCUSIÓN}

\subsection{Respuesta modelo constitutivo}

Las curvas esfuerzo-alargamiento de cada grupo, control (CTL) y tratado con melatonina (MEL), sobre las 
direcciones longitudinal y circunferencial y para un rango de análisis, $1 \leq \lambda \leq 2$, se muestran en la Figura 6 .

Con respecto a las curvas de ensayos individuales, los mayores esfuerzos se obtienen en el grupo control (CTL), donde tres (de cinco) muestras en la dirección circunferencial y dos (de cuatro) en la dirección circunferencial, superan los 200kPa. Por otra parte, el grupo MEL muestra menores esfuerzos (por debajo de $150 \mathrm{kPa}$ ) en ambas direcciones. Lo anterior significa que el tratamiento con melatonina reduce la rigidez de la arteria. En efecto, a partir de las Figuras 4 y 5 , se encuentran diferencias significativas entre grupos, en la mediana del esfuerzo uniaxial circunferencial de las curvas individuales. Para el grupo MEL, Mann-Whitney $U=16.7 \pm 0.4>U_{\text {crit }}\{5,4\}=16(0,10<p \leq 0,20)$, análisis de dos colas.

Por otra parte, las curvas ajustadas a la información experimental promedio, indicadas la Figura 6, permiten capturar el efecto de la disposición preferencial de las fibras de colágeno en el vaso, i.e. la influencia del ángulo $\varphi$, sobre la rigidez relativa - longitudinal versus circunferencial - del tejido.

En el grupo MEL, dado que $\varphi / 45 \mathrm{deg}=0,78$, se tiene una mayor rigidez en tracción longitudinal. Por ejemplo, para un alargamiento $\lambda=1.50$, se obtiene un esfuerzo $\sigma_{z} \sim 45 \mathrm{kPa}$, un $50 \%$ superior al esfuerzo circunferencial, $\sigma_{\theta} \sim 30 \mathrm{kPa}$. En contraste, dado que en el grupo control (CTL), $\varphi / 45 \mathrm{deg}=1.09$, se obtiene una mayor rigidez circunferencial; ídem alargamiento se asocia a un esfuerzo, $\sigma_{z} \sim 38 \mathrm{kPa}$ en la dirección longitudinal, aproximadamente un $10 \%$ inferior al esfuerzo en la dirección circunferencial, $\sigma_{\theta} \sim 42 \mathrm{kPa}$.

\subsection{Simulación numérica ensayo de acopado hidráulico}

Con respecto a la simulación numérica del ensayo de acopado hidráulico, las curvas de la Figura 7 muestran las discrepancias en el espesor mínimo normalizado de la estructura, entre ambos grupos (CTL y MEL), debidas a diferencias en el desplazamiento vertical del punto inferior (B); reflejando la dependencia de éste con las propiedades mecánicas del material para una presión superior a $5 \mathrm{kPa}$. En particular, el grupo MEL es más flexible que el grupo CTL, reafirmando un posible efecto del fármaco en las propiedades mecánicas.

En relación a la respuesta inelástica del material, las Figuras 7 y 8 muestran el efecto del daño con respecto al caso elástico, como un aumento en el desplazamiento vertical y en la tasa de liberación de energía, con respecto al caso elástico; ambos efectos consecuencia de a un aumento en la deformación.

Con respecto a la respuesta del ensayo, en la Figura 8 se aprecia la evolución del daño y de la tasa de liberación de energía, sobre la arista de interés (punto A). Las simulaciones predicen una intensidad de la presión para el inicio de daño, 22,0kPa para el grupo CTL, y 18,0kPa para el grupo MEL; asociados respectivamente, a los espesores mínimos de la pared, 1,32 mm (CTL) y 1,61 mm (MEL), correspondientes a un 66\% y un $70 \%$ del espesor inicial, respectivamente.

A modo referencial, la presión arterial media de los corderos es $\sim 11 \mathrm{kPa}(\sim 80 \mathrm{mmHg})$ [40]. Además, la presión intravascular (diastólica sistólica) del ser humano es aproximadamente, $12 \sim 16 \mathrm{kPa}(90 \sim$ $120 \mathrm{mmHg}$ ), y el umbral para una crisis de hipertensión, $16 \sim 24 \mathrm{kPa}(120 \sim 180 \mathrm{mmHg})$ [35]; por lo cual los valores entregados por las simulaciones son compatibles - i.e. comparten orden de magnitud - con un escenario de crisis de hipertensión mixta en humanos, validando la composición multiplicativa del daño, propuesta en Ecuación (8).

Finalmente, el tratamiento con melatonina disminuye la rigidez y mejora la capacidad elástica de la aorta torácica de corderos, en concordancia con estudios [42- 45] que reportan este efecto en otras arterias animales, corroborando nuestros resultados. Esto podría ser de utilidad en casos de problemas cardiovasculares que cursen con aumentos de la rigidez arterial, como la hipertensión sistémica. Además, este efecto se sumaría al efecto hipotensor que se le ha descrito a melatonina en animales y en seres humanos [37, 41].

\section{CONCLUSIONES}

En este trabajo, se ha investigado el comportamiento mecánico de aortas recolectadas desde corderos hipóxicos, mediante un modelo de material hiperelástico con dos direcciones de isotropía transversal y un modelo de daño isótropo, evaluándose una posible alteración por el tratamiento con melatonina.

Nuestros hallazgos, muestran que la melatonina genera un efecto en las propiedades biomecánicas de la aorta torácica, marcada por una disminución de la rigidez y por diferencias en las direcciones de isotropía -disminución del ángulo de disposición de las fibras de colágeno; lo último verificable mediante medición directa (histológica) de este parámetro, tal como se ha realizado, por ejemplo, en [36].

Finalmente, se ha evaluado el desempeño de los modelos de material determinados, en una simulación mediante elementos finitos, del ensayo de acopado hidráulico; lográndose predecir espesores y presiones asociadas al inicio del daño, las últimas compatibles con un escenario de crisis de hipertensión mixta. 


\section{AGRADECIMIENTOS}

Los autores agradecen el apoyo de la Comisión Nacional Chilena de Investigación Científica y Tecnológica (CONICYT), a través de los proyectos FONDECYT 1151119 y 1170608, CONICYT PCHA/Doctorado Nacional/2014-21140988; y a la Vicerrectoría de Postgrado de la Universidad de Santiago de Chile.

\section{BIBLIOGRAFÍA}

[1] HUMPHREY, J., "Continuum biomechanics of soft biological tissues", Proceedings of the Royal Society A, 2002b, v. 175, pp. 1-44.

[2] RUIZ, F., Principios de urgencias, emergencias y cuidados críticos, Andalucía, Alhulia, 1999.

[3] JAIN, K., Textbook of Hyperbaric Medicine, Göttingen, Hogrefe-Verlag, 2009.

[4] BURTSCHER, M., et al., "Extreme Terrestrial Environments: Life in Thermal Stress and Hypoxia. A Narrative Review", Frontiers in Physiology, v. 9, pp. 572, 2018.

[5] TORRES-RAMOS, Y., et al., "La disfunción del eritrocito en la hipoxia tisular en pacientes con EPOC y su relación con estrés oxidativo", Revista del Instituto Nacional de Enfermedades Respiratorias, v. 22, n. 4, pp. 356-365, 2009.

[6] WANG, Z., CHESLER, N., "Role of collagen content and cross-linking in large pulmonary arterial stiffening after chronic hypoxia", Biomechanics and Modeling in Mechanobiology, v. 11, pp. 279-289, 2012.

[7] CHESTER, M., et al., "Cinaciguat, a soluble guanylate cyclase activator, augments cGMP after oxidative stress and causes pulmonary vasodilation in neonatal pulmonary hypertension", American journal of physiology, Lung cellular and molecular physiology, v. 301, n. 5, pp. L755-L764, 2011.

[8] RICART, A., et al., "Effects of Sildenafil on the Human Response to Acute Hypoxia and Exercise", High Altitude Medicine \& Biology, v. 6 n. 1, 2005.

[9] CHEN, Y., et al., "Atrial natriuretic peptide-dependent modulation of hypoxia-induced pulmonary vascular remodeling", Life Sciences, v. 79, n. 14, pp. 1357-1365, 2006.

[10] HOLZAPFEL, G., Nonlinear solid mechanics, Chichester, John Wiley \& Sons Ltd, 2000.

[11] HOLZAPFEL, G., et al., "A new constitutive framework for arterial wall mechanics and a comparative study of material models", Journal of Elasticity and the physical science of solids, v. 61 n. 1-3, pp. 1-48, 2000.

[12] CAÑAS, D., et al., "Fetal Growth Restriction Induces Heterogeneous Effects on Vascular Biomechanical and Functional Properties in Guinea Pigs (Cavia porcellus)", Frontiers in Physiology, v. 8, 144, 2007.

[13] HOLZAPFEL, G., "Biomechanics of soft tissue". In: The Handbook of Materials Behavior Models, v. 3, CIMNE, pp. 1049-1063, 2001.

[14] PEÑA, E., "On the Microstructural Modeling of Vascular Tissues". In: Computational and Experimental Biomedical Sciences: Methods and Applications, v. 21, Lecture Notes in Computational Vision and Biomechanics, Springer, pp. 19-47, 2015.

[15] GARCÍA-HERRERA, C., et al., "Modelling and numerical simulation of the human aortic arch under in vivo conditions", Biomechanics and Modeling in Mechanobiology, v. 12, 1143, 2013.

[16] KARIMI, A., et al., "Measurement of the circumferential mechanical properties of the umbilical vein: experimental and numerical analyses", Computer Methods in Biomechanics and Biomedical Engineering, v. 18, n. 13, pp. 1-9, 2014.

[17] TRICERRI, P., et al., "A numerical study of isotropic and anisotropic constitutive models with relevance to healthy and unhealthy cerebral arterial tissues", International Journal of Engineering Science, v. 101, pp. 126-155, 2016.

[18] CALVO, B., et al., "On modelling damage process in vaginal tissue", Journal of Biomechanics, v. 42, pp. 642-651, 2009.

[19] MUÑOZ, M., et al., "An experimental study of the mouse skin behavior: Damage and inelastic aspects", Journal of Biomechanics, v. 41, pp. 93-99, 2008.

[20] CALVO, B., et al., "An uncoupled directional damage model for fibered biological soft tissues. Formulation and computational aspects", International Journal for Numerical Methods in Engineering, v. 69, pp. 2036-2057, 2007. 
[21] PEÑA, E., et al., "On finite-strain damage of viscoelastic-fibered materials. Application to soft biological tissues", International Journal for Numerical Methods in Engineering, v. 74, pp. 1198-1218, 2008.

[22] PROVENZANO, P., et al., "Subfailure damage in ligament: a structural and cellular evaluation", Journal of Applied Physiology, v. 92 n. 1, pp. 362-371, 2002.

[23] BALZANI, D., et al., "Constitutive framework for the modeling of damage in collagenous soft tissues with application to arterial walls", Computer Methods in Applied Mechanics and Engineering, v. 213-216, pp. 139-151, 2012.

[24] MAHER, E., et al., "An anisotropic inelastic constitutive model to describe stress softening and permanent deformation in arterial tissue", Journal of the Mechanical Behavior of Biomedical Materials, v. 12, pp. 9-19, 2012a.

[25] PEÑA, E., "Computational aspects of the numerical modelling of softening, damage and permanent set in soft biological tissues", Computers and Structures, v. 130, pp. 57-72, 2014.

[26] PIERCE, D., et al., "Human thoracic and abdominal aortic aneurysmal tissues: Damage experiments, statistical analysis and consitutive modeling", Journal of the Mechanical Behavior of Biomedical Materials, v. 41, pp. 92-107, 2015.

[27] WEISBECKER, H., et al., "Layer-specific damage experiments and modeling of human thoracic and abdominal aortas with non-atherosclerotic intimal thickening", Journal of the Mechanical Behavior of Biomedical Materials, v. 12, pp. 93-106, 2012.

[28] GASSER, T., HOLZAPFEL, G., "A rate-independent elastoplastic model for biological fiber-reinforced composites at finite strains: continuum basis, algorithmic formulation and finite element implementation", Computational Mechanics 29, pp. 340-360, 2002.

[29] GASSER, T., et al., "Hyperelastic modelling of arterial layers with distributed collagen fibre orientations", Journal of the Royal Society Interface, v. 3, pp. 15-35, 2006.

[30] BALZANI, D., et al., "Simulation of discontinuous damage incorporating residual stresses in circumferentially overstretched atherosclerotic arteries", Acta Biomaterialia, v. 2, pp. 609-618, 2006.

[31] VOLOKH, K., VORP, D., "A model of growth and rupture of abdominal aortic aneurysm", Journal of Biomechanics, v. 41, pp. 1015-1021, 2008.

[32] ALTUN, A., UGUR-ALTUN B., "Melatonin: therapeutic and clinical utilization", International Journal of Clinical Practice, v. 61, n. 5, pp. 835-845, 2007.

[33] PEÑA, E., et al., "On the Mullins effect and hysteresis of fibered biological materials: A comparison between continuous and discontinuous damage models", International Journal of Solids and Structures, v. 46, pp. 1727-1735, 2009.

[34] KANZOW, C., et al., "Levenberg-Marquardt methods with strong local convergence properties for solving nonlinear equations with convex constraints", Journal of Computational and Applied Mathematics, v. 172, n. 2, pp. 375-397, 2004.

[35] REBOUSSIN, D., et al., "Systematic Review for the 2017 ACC/ AHA/ AAPA/ ABC/ ACPM/ AGS/ AphA/ ASH/ ASPC/ NMA/ PCNA Guideline for the Prevention, Detection, Evaluation, and Management of High Blood Pressure in Adults", Journal of the American College of Cardiology, v. 71, pp. 2176-2198, 2018.

[36] HORNY, L., et al., "A distribution of collagen fiber orientations in aortic histological section", In: XII Mediterranean Conference on Medical and Biological Engineering and Computing, pp. 772-775, Calcídica, Grecia, Mayo 2010.

[37] ASTORGA, C., et al., "Melatonin Decreases Pulmonary Vascular Remodeling and Oxygen Sensitivity in Pulmonary Hypertensive Newborn Lambs", Frontiers in physiology, v. 9, pp. 185, 2018.

[38] TORRES, F., et al., "Melatonin reduces oxidative stress and improves vascular function in pulmonary hypertensive newborn sheep", Journal of pineal research, v. 58, n. 3, pp. 362-373, 2015.

[39] GONZÁLEZ-CANDIA, A., et al., "Melatonin long-lasting beneficial effects on pulmonary vascular reactivity and redox balance in chronic hypoxic ovine neonates", Journal of pineal research, v. 68, n. 1, pp. e12613, 2020.

[40] HERRERA, E., et al., "High-altitude chronic hypoxia during gestation and after birth modifies cardiovascular responses in newborn sheep", American Journal of Physiology-Regulatory, Integrative and Comparative Physiology, v. 292, n. 6, pp. R2234-R2240, 2007.

[41] BAKER, J., KIMPINSKI, K., "Role of melatonin in blood pressure regulation: An adjunct antihypertensive agent", Clinical and Experimental Pharmacology and Physiology, v. 45, n. 8, pp. 755-766, 
2018.

[42] TARE M., et al., "Maternal melatonin administration mitigates coronary stiffness and endothelial dysfunction and improves heart resilience to insult in growth restricted lambs", Journal of Physiology, v. 592, n. 12, pp. 2695-2709, 2014.

[43] PRUSA A., PLASS, C., "Melatonin attenuates thiocyanate-induced vasoconstriction in aortic rings", Saudi Pharmaceutical Journal, v. 25, n. 7, pp. 993-998, 2017.

[44] GROSSMAN E., et al., "Effect of melatonin on nocturnal blood pressure: meta-analysis if randomized controlled trials", Vascular Health and Risk Management, v. 7, pp. 577-584, 2011.

[45] REPOVA-BEDNAROVA K., et al., "Effect of Captopril and Melatonin on Fibrotic Rebuilding of the Aorta in 24 Hour Light-Induced Hypertension”, Physiological Research, v. 62, pp. S135-S141, 2013.

\section{ORCID}

Alejandro Bezmalinovic

Eugenio Rivera

Claudio García-Herrera

Diego Celentano

Alejandro González-Candia

Emilio A. Herrera
https://orcid.org/0000-0002-2698-770X

https://orcid.org/0000-0001-8103-2706

https://orcid.org/0000-0002-1283-8551

https://orcid.org/0000-0002-7600-0619

https://orcid.org/0000-0001-8429-367X

https://orcid.org/0000-0002-6342-085X 